\title{
THE DISTRIBUTION OF MAGNETIZATION FOR A STEPPED FERRO-ANTIFERROMAGNET INTERFACE
}

\author{
O.K. Dudko and A.S. Kovalev \\ B. Verkin Institute for Low Temperature Physics and Engineering \\ Ukrainian Academy of Sciences, 47 Lenin Avenue, 310164 Kharkov, Ukraine
}

Magnetization distribution of a disclination type connected with the atomic step on the ferro-antiferromagnet boundary is studied. The results are generalized for the periodic stepped interface.

PACS numbers: $75.30 . \mathrm{Kz}$

Multilayer systems with alternating layers of ferromagnets (FM) and antiferromagnets (AFM) [1] have been drawing a special attention. In experiments some interlayers are often wedge-shaped with a small divergence angle [2] and the interface has a stepped form with a large separation between steps. The presence of a step at the boundary of a layered AFM leads to local variation of the magnetic structure [3]. We shall show that the presence of an atomic step at the FM-AFM interface leads to the emergence of the magnetic disclination.

Let us consider a plane FM-AFM interface with a strong easy-plane anisotropy. All spins are characterized only by their angle in this plane. In the presence of an atomic size step at this boundary, the spin distribution is noncollinear and the system acquires a magnetic disclination having Frank's index $k=1$ and its center at a step. We replace an interface with a step by the ideal boundary with the reversed sign of the exchange interaction through it on one of the semi-axes.

Let us consider a 2D lattice with the FM-AFM interface along the $x$-axis, antiferromagnetic ordering along $z$-direction and the Hamiltonian

$$
H=\Sigma_{n m} J_{n m} \cos \left(\phi_{n}-\phi_{m}\right),
$$

where $\phi_{n}$ is the spin rotation angle in the $(x, z)$ plane and $J_{n m}$ the exchange interaction between the nearest spins. In the FM half-space $(z>0) J_{n m}=-J_{1}$ and $J_{n m}=-J_{2}$ in the $x$ - and $z$-directions, while in the AFM $(z<0), J_{n m}=-\tilde{J}_{1}$ and $\tilde{J}_{2}$, in these directions, where $J_{i}, \tilde{J}_{i}>0$. We propose $J_{n m}=J_{\mathrm{s}} \operatorname{sgn}(x)$ for exchange interaction through the interface. It is convenient to change from angles $\phi_{n}$ to $\phi_{n}+\pi$ in even-numbered layers of AFM. After this we can provide a longwave description in both half-spaces

$$
J_{1} \phi_{x x}+J_{2} \phi_{z z}=0 \quad(z>0), \quad \tilde{J}_{1} \phi_{x x}+\tilde{J}_{2} \phi_{z z}=0 \quad(z<0)
$$

and the interaction between FM and AFM must be taken exactly 


$$
W_{\mathrm{s}}=\operatorname{sgn}(x) J_{\mathrm{s}} \cos \left(\phi_{+}-\phi_{-}\right),
$$

where $\phi_{ \pm}=\phi(z= \pm 0)$. The solution of the Neuman problem for the scalar field $\phi(x, z)$ in the half-spaces $(z>0)$ and $(z<0)$ produces the following relation between $\mathrm{d} \phi_{+} / \mathrm{d} x$ and $\partial \phi /\left.\partial z\right|_{z=+0}$ at the surface:

$$
\left(J_{1} / J_{2}\right)^{1 / 2} \mathrm{~d} \phi_{+} / \mathrm{d} x=\widehat{H} \partial \phi /\left.\partial z\right|_{z=+0},\left(\tilde{J}_{1} / \tilde{J}_{2}\right)^{1 / 2} \mathrm{~d} \phi_{-} / \mathrm{d} x=\widehat{H} \partial \phi /\left.\partial z\right|_{z=-0},
$$

where $\widehat{H}$ is the Hilbert transform $\widehat{H} F=(1 / \pi) \int \mathrm{d} x^{\prime} F\left(x^{\prime}\right) /\left(x-x^{\prime}\right)$.

The dependence of the "forces" $J_{2} \partial \phi /\left.\partial z\right|_{z=+0}$ and $-\tilde{J}_{2} \partial \phi /\left.\partial z\right|_{z=-0}$ on the relative orientation of spins at the FM-AFM boundary follows from (3). After adding and subtracting Eqs. (4), we obtain a closed equation for $\psi=\phi_{+}-\phi_{-}$and the relation of the functions $\phi_{ \pm}$with $\psi(x)$

$$
\begin{aligned}
& \sin \psi+l \operatorname{sgn}(x) \hat{H}(\mathrm{~d} \psi / \mathrm{d} x)=0, \\
& \phi_{+}=\left[\left(\tilde{J}_{1} \tilde{J}_{2}\right)^{1 / 2} /\left[\left(J_{1} J_{2}\right)^{1 / 2}+\left(\tilde{J}_{1} \tilde{J}_{2}\right)^{1 / 2}\right]\right] \psi, \phi_{-}=-\left(J_{1} J_{2} / \tilde{J}_{1} \tilde{J}_{2}\right)^{1 / 2} \phi_{+},
\end{aligned}
$$

where $l=\left(J_{1} J_{2} \tilde{J}_{1} \tilde{J}_{2}\right)^{1 / 2} / J_{\mathrm{s}}\left[\left(J_{1} J_{2}\right)^{1 / 2}+\left(\tilde{J}_{1} \tilde{J}_{2}\right)^{1 / 2}\right]$.

Equation (5) was studied in Ref. [4]. It has a soliton solution with the asymptotics

$$
\psi(\kappa) \simeq\left\{\begin{array}{l}
1 / \kappa \quad(\kappa \rightarrow-\infty) \\
\pi / 2-(2 / \pi) \ln |\kappa| \quad(\kappa \ll 1) \\
\pi-1 / \kappa \quad(\kappa \rightarrow+\infty),
\end{array}\right.
$$

where $\kappa=x / l$. Spin distributions at the FM-AFM boundary and in the bulk are expressed in terms of $\psi(\kappa)$. Thus, spin rotations in layers adjoining the interface are defined by $(6)$. The boundary region in which the spin angles are not small is of the order of $l$. A decrease in the interaction through the boundary leads to an increase in the size of the disordered region in proportion to $1 / J_{\mathrm{s}}$. The total rotation of spins in the corresponding half-spaces are $\delta \phi_{+}=\phi_{+}(+\infty)-\phi_{+}(-\infty)=$ $\pi /(1+g), \delta \phi_{-}=-\pi g /(1+g)$, where $g=\left(J_{1} J_{2} / \tilde{J}_{1} \tilde{J}_{2}\right)^{1 / 2}$. The total variation of $\phi$ during circumvention of the step is $\pi$, and this spin configuration corresponds to magnetic disclination with the index $k=1$.

If magnetic interaction in the AFM is much stronger than in the FM $\left(\tilde{J}_{i} \gg J_{i}\right)$, the rotation of magnetization occurs mainly in the FM region through. an angle close to $\pi$. In this case, even a weak anisotropy in the easy plane leads to a formation of a domain wall in the FM which ends at the step in the interface. In the case of a "frozen" FM $\left(J_{i} \gg \tilde{J}_{i}\right)$, the spins rotate mainly in the AFM, and a $180^{\circ}$ AFM-domain wall is formed if the in-plane anisotropy is taken into account.

The field distribution around the step depends on the effective forces obtained from (3). We are interested in the asymptotics of the solution at distances $\kappa \gg 1$, so approximate $\sin \left(\phi_{+}-\phi_{-}\right)$in the relation for the "forces" by the expression $1 /\left(1+\kappa^{2}\right)^{1 / 2}$. Then for $x \gg l$ and $z \gg \lambda$, where $\lambda=l\left(J_{1} / J_{2}\right)^{1 / 2}$, we have

$$
\begin{gathered}
\partial \phi / \partial z(x, z>0) \simeq\left(J_{\mathrm{s}} / J_{2}\right) \frac{(x / l)}{\left(x^{2} / l^{2}+z^{2} / \lambda^{2}\right)} \\
\times\left\{1-4|z| /\left[\lambda \pi\left(x^{2} / l^{2}+z^{2} / \lambda^{2}\right)\right]\right\} .
\end{gathered}
$$


The expression for the AFM has a similar form when replacing $J_{i} \longleftrightarrow \tilde{J}_{i}$. For $J_{1} \simeq J_{2}$ and $\tilde{J}_{1} \simeq \tilde{J}_{2}$ we have $[\partial \phi / \partial z(z>0)] /[\partial \phi / \partial z(z<0)] \approx \tilde{J}_{2} / J_{2}$, and the spin distribution is quite asymmetric in the case of a considerable difference between the exchange constants in FM and AFM.

Let us generalize the obtained results to the case of an interface with an infinite number of atomic size steps with a large separation $L \gg a$. The exchange interaction through the boundary can now be described by the following function:

$$
W=J_{\mathrm{s}}(x) \cos \left(\phi_{+}-\phi_{-}\right),
$$

where $J_{\mathrm{s}}(x)$ has the form of an alternating steps function with a period $2 L$ and amplitude $J_{\mathbf{s}}$, characterizing the interaction between the half-spaces. Using Eqs. (4), we obtain an equation analogous to (5) for $\psi$ and the relation between $\phi_{+}$and $\phi_{-}$

$$
\left(J_{\mathrm{s}}(x) / J_{\mathrm{s}}\right) \sin \psi+l \hat{H} \mathrm{~d} \psi / \mathrm{d} x=0, \quad \phi_{-}=-g \phi_{+} .
$$

To analyze the solution of Eq. (10) qualitatively we replace our system by a model piecewise linear system with the interface interaction

$$
W_{\mathrm{s}} \longrightarrow\left\{\begin{array}{l}
J_{\mathrm{s}} \psi^{2} / 2-J_{\mathrm{s}}, \quad 0 \leq \psi<\pi / 2 \\
-J_{\mathrm{s}}(\psi-\pi)^{2} / 2+J_{\mathrm{s}}, \quad \pi / 2 \leq \psi \leq \pi
\end{array}\right.
$$

In Eq. (10), $\sin \psi$ is replaced by a piecewise linear function

$$
\sin \psi \longrightarrow\left\{\begin{array}{l}
\psi, \quad((2 n-1) L<x<2 n L), \\
\pi-\psi, \quad(2 n L<x<(2 n+1) L),
\end{array}\right.
$$

where $n$ is an integer. We shall consider below the ground state of the system in which magnetization distribution becomes uniform at large distances from the interface. It corresponds to a regular alternation of the signs of magnetic disclinations associated with the surface steps.

Weak bond between FM and AFM. If the size of the inhomogeneity is comparable with the separation between steps the solution for $\psi$ to a first approximation is

$\psi \simeq \pi / 2+2 \sin (\pi x / L) /(1+l \pi / L)$.

The spin deviations near the interface are obtained from $(10,13)$

$$
\phi_{+} \simeq \frac{\pi / 2}{1+\left(J_{1} J_{2} / \tilde{J}_{1} \tilde{J}_{2}\right)^{1 / 2}}+\frac{2}{1+\left(J_{1} J_{2} / \tilde{J}_{1} \tilde{J}_{2}\right)^{1 / 2}} \frac{\sin \pi x / L}{1+l \pi / L}, \phi_{-}=-g \phi_{+} .
$$

It follows from (14) that for a weak bond between the half-spaces, the spin deviation in the boundary layers is weakly modulated. Spin configuration is determined by the relation between $J_{i}$ and $\tilde{J}_{i}$. When the exchange interaction in the magnets is identical $\left(\tilde{J}_{1} \tilde{J}_{2}=J_{1} J_{2}\right)$, the angle between mean directions of spins in the FM and AFM is $\pi / 2$, and the amplitude of spin deviations from these directions is $\left(1+\pi \sqrt{J_{1} J_{2}} / 2 L J_{\mathrm{s}}\right)^{-1}$. If the interaction in AFM is considerably stronger than in FM $\left(\tilde{J}_{1} \tilde{J}_{2} \gg J_{1} J_{2}\right)$, spins rotate mainly in the FM region and deviate periodically from the mean direction orthogonal to the uniform orientation of spins in the AFM. In the case of a "frozen" FM, the spin rotation mainly occurs in the AFM region. 
Using (4) after the Hilbert transform and substituting the obtained expressions (14) we arrive at an expression for derivative of magnetization

$$
\begin{aligned}
& \partial \psi / \partial z(x, z>0) \\
& \quad=(2 \pi / L)\left(J_{1} / J_{2}\right)^{1 / 2} \frac{\exp \left[(-\pi z / L)\left(J_{1} / J_{2}\right)^{1 / 2}\right] \sin (\pi x / L)}{\left[1+\left(J_{1} J_{2} / \tilde{J}_{1} \tilde{J}_{2}\right)^{1 / 2}(1+l \pi / L)\right.} .
\end{aligned}
$$

The expression for AFM differs from (15) by replacing $J_{i} \longleftrightarrow \tilde{J}_{i}$.

The characteristic size of the nonuniform region near the FM-AFM boundary is of the order of $L \sqrt{J_{2} / J_{1}}$ in the FM and $L \sqrt{\tilde{J}_{2} / \tilde{J}_{1}}$ in the AFM. Nonuniformity in the magnetization distribution upon a weak interaction through the interface is proportional to $1 / l \propto J_{\mathrm{s}}$ and vanishes for $J_{\mathrm{s}}=0$. Magnetization distribution with coordinate $z$ depends only on the properties of the half-space and not on $J_{\mathbf{s}}$.

Strong bond between $F M$ and $A F M$. In this limit let us first consider the case when AFM is "frozen". If the spins in the AFM are collinear to the interface, strong interaction through it "aligns" the spins in the FM along the interface. A transition in the upper half-space through the step along the $x$-axis turns the spins through an angle $\pi$. In the general case of "defrozen" magnets, it is obvious that, for a strong bond between them, the spins rotate through an angle $\pi / 2$ in each half-space upon a change in $x$ by $L$, while the relative spin deviation varies between 0 and $\pi$. The derivatives of spin deviations near the boundary are

$$
\partial \phi_{ \pm} / \partial x= \pm(\pi / 2) \sum_{-\infty}^{\infty} \delta(x-n l)(-1)^{n}
$$

Then the distribution of the field in the FM at $z \gg L \sqrt{J_{2} / J_{1}}$ is

$$
\begin{aligned}
& \partial \phi / \partial z(x, z>0) \\
& \quad \simeq\left(\pi^{2} / 2 L\right)\left(J_{1} / J_{2}\right)^{1 / 2} \sin (\pi x / L) \exp \left[-z\left(J_{1} / J_{2}\right)^{1 / 2} \pi / L\right] .
\end{aligned}
$$

It can be seen from (17) that the decrease in the nonuniform magnetization into the bulk is exponential and the region of such a distribution has a size $\Delta_{\mathrm{FM}} \sim$ $L \sqrt{J_{1} / J_{2}}$ in the FM and $\Delta_{\mathrm{AFM}} \sim L \sqrt{\tilde{J}_{1} / \tilde{J}_{2}}$ in the AFM.

\section{References}

[1] R.L. Stamps, R.E. Camley, Phys. Rev. B 54, 15200 (1996).

[2] D. Weller, A. Carl, in: Colloquium Digest of 14th International Colloquium on Magnetic Films and Surfaces, Düsseldorf, Germany, Heinrich-Heine Universität, Düsseldorf 1994, p. 545 .

[3] S. Blügel, D. Pescia, P.H. Dederichs, Phys. Rev. B 39, 1392 (1989).

[4] O.K. Dudko, A.S. Kovalev, Low Temp. Phys. 24, 422 (1998). 